\title{
Adición de metil- $\beta$-ciclodextrina cargada de colesterol en la criopreservación de semen de carnero
}

\author{
Addition of cholesterol-loaded methyl- $\beta$-cyclodextrin in cryopreservation \\ of ram semen
}

\author{
Luis Antonio Castillo Cevallos ${ }^{1}$, Enrique Páucar Espinoza ${ }^{2,4}$, \\ Enrique Alvarado Malca $^{3}$
}

\section{Resumen}

Se evaluó el efecto de la adición de metil- $\beta$-ciclodextrina cargada de colesterol (CLC) sobre las características microscópicas del semen refrigerado/descongelado de carnero. Se emplearon cuatro carneros Asblack de 1-2 años. Se usaron 30 eyaculados divididos en grupos de tres alícuotas a los que se agregó CLC ( 0,1 y 2 mg de CLC/120 millones de espermatozoides, T1, T2 y T3, respectivamente) a $22^{\circ} \mathrm{C}$ y fueron incubados por $15 \mathrm{minu}$ tos. Las muestras luego fueron refrigeradas y estabilizadas a $5{ }^{\circ} \mathrm{C}$ durante 18 horas. El congelamiento del semen se hizo en pajillas usando vapores de nitrógeno por 4 minutos $\left(-120^{\circ} \mathrm{C}\right)$ y luego sumergidas en nitrógeno líquido. No hubo diferencias estadísticas entre las medias de los tratamientos en todas las características del semen refrigerado. Los resultados pos-descongelación demuestran mejoras con el uso de colesterol en la criopreservación de semen de carnero $(\mathrm{p}<0.5)$, obteniendo una motilidad de $65.3,68.7 \mathrm{y}$ $75.37 \%$, vitalidad de $56.6,60.7$ y $66.2 \%$ e integridad de membrana de $50.5,56.4$ y $63.7 \%$, para T1, T2 y T3, respectivamente. No hubo diferencia estadística entre tratamientos con respecto a las anormalidades espermáticas.

Palabras clave: criopreservación; semen; ciclodextrina; colesterol; carnero

\section{AbSTRACT}

The effect of the addition of cholesterol-loaded methyl- $\beta$-cyclodextrin (CLC) on the microscopic characteristics of chilled and frozen/thawed ram semen was evaluated. Four 1-2-year-old Asblack rams were used. Thirty ejaculates divided into groups of three

${ }^{1}$ Facultad de Zootecnia, Universidad Nacional Agraria La Molina, Lima, Perú

${ }^{2}$ Banco Nacional de Semen, Universidad Nacional Agraria La Molina, Lima, Perú

${ }^{3}$ Departamento de Producción Animal, Facultad de Zootecnia, Universidad Nacional Agraria La Molina, Lima, Perú

${ }^{4}$ E-mail: epaucar@lamolina.edu.pe

Recibido: 19 de enero de 2019

Aceptado para publicación: 1 de octubre de 2019 
aliquots each were used to which CLC ( 0,1 and $2 \mathrm{mg}$ of CLC/120 million sperm, T1, T2 and $\mathrm{T} 3$ respectively) were added at $22{ }^{\circ} \mathrm{C}$ and incubated for 15 minutes. Samples were then refrigerated and stabilized at $5{ }^{\circ} \mathrm{C}$ for 18 hours. Semen freezing was done in straws using nitrogen vapours for 4 minutes $\left(-120^{\circ} \mathrm{C}\right)$ and then submerged in liquid nitrogen. There were no statistical differences between treatment averages in all characteristics of refrigerated semen. The post thawing results showed improvements with the use of cholesterol in the cryopreservation of ram semen $(p<0.5)$, obtaining a motility of $65.3,68.7$ and $75.37 \%$, vitality of $56.6,60.7$ and $66.2 \%$ and membrane integrity of $50.5,56.4$ and $63.7 \%$ in $\mathrm{T} 1, \mathrm{~T} 2$ and $\mathrm{T} 3$ respectively. There was no statistical difference between treatments with respect to sperm abnormalities.

Key words: cryopreservation; semen; cyclodextrin; cholesterol; ram

\section{INTRODUCCIÓN}

El 81\% de la población ovina en el Perú es de tipo criollo y el $94 \%$ del total de la población se encuentra en la zona de sierra (SENAGRO, 2012), mayormente a cargo de las comunidades campesinas, que no cuentan con programas de mejora genética (FAO, 2004), lo cual se podría realizar con apoyo de la inseminación artificial con semen congelado vía laparoscópica (Eppleston y Maxwell, 1993). Sin embargo, la calidad del semen congelado de ovinos es baja con relación al semen fresco, determinando bajas tasas de preñez $(<40 \%)$ (Watson, 2000). No obstante, con la inseminación intrauterina laparoscópica se puede conseguir tasas de concepción aceptables (Anel et al., 1992).

Los espermatozoides criopreservados sufren daños bioquímicos (ralentización metabólica) y estructurales (cambios en la estructura de las bicapas lipídicas de las membranas, tanto plasmáticas como de los orgánulos), que finalmente afectan la funcionalidad de los espermatozoides y su capacidad fecundante. Los cambios osmóticos y la formación de hielo intracelular son las principales causas de estos daños celulares (Holt, 2000).

Diversos estudios demuestran que la adición de ciclodextrinas cargadas de colesterol al semen puede incrementar eficien- temente la funcionalidad de la criopreservación de los espermatozoides de varias especies de interés (Purdy y Graham, 2004; Moore et al., 2005; Mocé et al., 2010b; Fashard et al., 2001; Tomás et al., 2014). Para la adición de colesterol se pueden utilizar oligosacáridos cíclicos, denominados ciclodextrinas, los cuales poseen una cara hidrofílica externa y una interna hidrofóbica (Mocé et al., 2010b). Las ciclodextrinas pueden cargarse con colesterol antes de su utilización, y de esta manera van a servir como medio transportador para incorporarlo a las membranas plasmáticas de los espermatozoides previo a la congelación, mejorando así su fluidez y estabilidad ante los cambios osmóticos y de temperatura (Mocé et al., 2010b).

En base a los antecedentes mencionados, el objetivo de esta investigación fue evaluar el efecto de la adición de metil- $\beta$ ciclodextrina cargada con colesterol sobre la calidad de las características microscópicas del semen de carnero refrigerado y descongelado.

\section{Materiales y Métodos}

\section{Lugar del Estudio}

El presente trabajo de investigación se realizó en las instalaciones del Banco Nacional de Semen del Programa de Mejoramien- 
to Animal de la Universidad Nacional Agraria la Molina (UNALM), ubicado en el distrito de la Molina, Lima, Perú. El clima presenta temperaturas mínimas mensuales promedio de $16.2{ }^{\circ} \mathrm{C}$ y máximas de $22.5^{\circ} \mathrm{C}$, humedad relativa promedio anual de $81 \%$ y precipitación total anual de $10 \mathrm{~mm}$.

\section{Animales}

Se utilizaron cuatro carneros Asblack (3/4 Assaf - 1/4 Black Belly) con edades entre 1 y 2 años, pertenecientes al centro pecuario Rigoranch de la UNALM. Los animales permanecieron bajo un mismo régimen de alimentación, manejo y cuidado sanitario. Fueron alimentados con forraje verde, maíz chala picado, un suplemento a base de concentrado y agua ad libitum. Los carneros se mantuvieron en corrales de tierra de $2.8 \mathrm{x}$ $3.0 \times 1.2 \mathrm{~m}$.

\section{Ciclodextrinas Cargadas de Colesterol (CLC)}

Los productos químicos utilizados para la elaboración de las ciclodextrinas cargadas de colesterol fueron de Sigma-Aldrich (Cód. C8667-5G). La solución de CLC fue preparada según lo descrito por Purdy y Graham (2004). Brevemente, en un tubo de ensayo se disolvieron $200 \mathrm{mg}$ de colesterol en $1 \mathrm{ml}$ de cloroformo. En otro tubo de ensayo se diluyó $1 \mathrm{~g}$ de metil- $\beta$-ciclodextrina (SigmaAldrich, Cód. C4555-5G) en $2 \mathrm{ml}$ de metanol, se adicionaron $450 \mu \mathrm{l}$ de la solución de colesterol y homogenizó. En una caja Petri se removió el solvente usando una corriente de nitrógeno gaseoso. Los cristales resultantes se dejaron secar $24 \mathrm{~h}$, se pasaron a un recipiente de vidrio y se almacenaron a $22{ }^{\circ} \mathrm{C}$ hasta su uso. La solución de CLC se preparó disolviendo $50 \mathrm{mg}$ de cristales CLC a $1 \mathrm{ml} \mathrm{de}$ medio de dilución Tris-cítrico-glucosa $(3.637 \mathrm{~g}$ Tris, $1.99 \mathrm{~g}$ ácido cítrico, $1.50 \mathrm{~g}$ glucosa) y $100 \mathrm{ml}$ de agua desionizada y esterilizada, $\mathrm{pH}$ próximo a 7 y osmolaridad cercana a 330 $\mathrm{mOsm} / 1$, se mezcló con un vórtex para homogenizar se incubó en baño maría a $37{ }^{\circ} \mathrm{C}$ hasta su uso.

\section{Evaluación del Semen}

Se realizaron colecciones semanales considerando un régimen de dos colecciones por día, seguido de un descanso de dos días por macho. El semen fue colectado por medio de vagina artificial utilizando una hembra para simular la monta. Se determinó el volumen, color y $\mathrm{pH}$ en las muestras inmediatamente después de la colección.

La evaluación de las características microscópicas del semen fue realizada en tres momentos: la primera con semen fresco, inmediatamente de obtenida la muestra y en estado de equilibrio; la segunda una vez que la muestra estuvo diluida con AndroMed ${ }^{\circledR}$, refrigerada y equilibrada a $5{ }^{\circ} \mathrm{C}$, antes de ser congelada; y la tercera luego de la descongelación. En todos los casos de determinó la motilidad, vitalidad, anormalidades y funcionalidad de membrana espermática, y en fresco se determinó, además, la concentración espermática.

\section{Concentración y motilidad espermática}

La concentración y la motilidad fueron determinadas utilizando el sistema de Análisis Computarizado de Semen (CASA) Andro Vision ${ }^{\circledR}$ (Minitube). Para el primer caso, se colocó una gota de $3 \mu 1$ de la muestra de semen diluida (1:49) con diluyente comercial AndroMed ${ }^{\circledR}$ en una cámara de recuento espermático. Para evaluar la motilidad, expresada en porcentaje, se colocaron $6 \mu 1$ de la muestra de semen sobre una lámina portaobjetos, previamente temperada a $37^{\circ} \mathrm{C}$ y se cubrieron con una laminilla cubreobjetos temperada. En ambos casos, las muestras se observaron con un objetivo de $10 \mathrm{X}$ en un microscopio de contraste de fases y los análisis se hicieron con el módulo de Motilidad y Concentración del software AndroVision ${ }^{\circledR}$.

\section{Vitalidad espermática}

Para el conteo de los espermatozoides vivos se utilizaron $5 \mu \mathrm{l}$ de semen puro, $5 \mu \mathrm{lde}$ eosina y $5 \mu 1$ de nigrosina. La mezcla se 
homogenizó y se hizo un frotis en una lámina portaobjeto dejándolo a temperatura ambiente de laboratorio por 20 segundos, para luego ser observados al microscopio con un aumento de 40x. Se hizo el conteo de 200 células, determinándose la cantidad de espermatozoides vivos (células blancas) y espermatozoides muertos (células de color rojo). El resultado fue expresado en porcentaje.

\section{Anormalidades espermáticas}

Se utilizó la misma lámina empleada para evaluar la vitalidad y en el microscopio a 40x. Se realizaron tres conteos por muestra y en cada uno se contabilizaron 200 espermatozoides. El resultado fue expresado en porcentaje.

$\begin{aligned} & \text { Funcionalidad de la membrana } \\ & \text { espermática }\end{aligned}$

Se utilizó la prueba de HOST (Hipo Osmotic Swelling Test), que consiste en la exposición de los espermatozoides a un medio hiposmótico, según el protocolo de Correa y Zavos (1994), mezclando el semen con solución hiposmótica a $100 \mathrm{mOsmol} / 1$, en una proporción de $1: 10$. Se incuba en baño maría a $34{ }^{\circ} \mathrm{C}$ por $60 \mathrm{~min}$ y luego se procede a mezclar una gota de la muestra seminal con una gota de glutaraldehido al $2 \%$ para retener la reacción. Se contaron 200 espermatozoides en el microscopio de contraste de fase a $40 \mathrm{X}$. Se consideraron espermatozoides con membrana funcional $(+)$ los que reaccionaron al estrés hiposmótico mediante la hinchazón de la parte distal de la cola espermática.

\section{Procesamiento de Semen}

Se utilizó una parte del dilutor Andromed ${ }^{\circledR}$ (Minitube) y 4 partes de agua bidestilada en baño maría a $33.5^{\circ} \mathrm{C}$ por 15 minutos para luego mezclarlo y homogenizarlo. El semen fue meclado con el dilutor en una proporción de 1/1. Luego, las muestras de semen se dividieron en tres alícuotas (control sin CLC, $1 \mathrm{mg}$ de CLC y $2 \mathrm{mg}$ de
CLC por 120 millones de espermatozoides), agregándose la fracción requerida a cada alícuota. Se incubaron por 15 minutos a temperatura ambiente de laboratorio $\left(22^{\circ} \mathrm{C}\right)$, como describe Purdy y Graham (2004). El semen diluido fue envasado pajillas Cassou (IMV Technologies, Francia) de $0.5 \mathrm{ml}$ en concentración de 60 millones de espermatozoides $/ \mathrm{ml}$. Las pajillas fueron selladas con alcohol polivinílico.

Para la congelación, se inició el descenso de la temperatura de las pajillas en una refrigeradora, llevando el semen desde $22^{\circ} \mathrm{C}$ hasta $5{ }^{\circ} \mathrm{C}$ en 2 horas, aproximadamente, y se estabilizó en la refrigeradora a $5^{\circ} \mathrm{C}$ durante $18 \mathrm{~h}$, según las recomendaciones de Quispe (1998). Posteriormente, las pajillas se congelaron en vapores de nitrógeno líquido $\left(-120^{\circ} \mathrm{C}\right)$ en un tiempo de 4 minutos y luego se sumergieron directamente en el nitrógeno líquido. El descongelamiento de las pajillas se realizó a una temperatura de $38{ }^{\circ} \mathrm{C}$ en un termo descongelador por 20-30 segundos.

\section{Análisis Estadístico}

Para las características macro y microscópicas del semen fresco se utilizó estadística descriptiva (promedios y desviaciones estándar). Los datos porcentuales se transformaron angularmente por medio del arcoseno para acercarlos a una distribución normal. Para la determinación del efecto de las ciclodextrinas cargadas de colesterol sobre las variables evaluadas se realizó un análisis de Diseño en Bloques Completamente al Azar con submuestreos. Para la comparación múltiple de medias se aplicó la prueba de Tukey.

\section{Resultados y Discusión}

\section{Viabilidad del Semen Refrigerado}

No se encontraron diferencias significativas entre tratamientos para las variables en estudio (Cuadro 1). 
Cuadro 1. Características del semen refrigerado de carnero y tratado con metil- $\beta$ ciclodextrinas saturadas de colesterol (CLC/120 x $10^{6}$ espermatozoides)

\begin{tabular}{lccc}
\hline Variable & $0 \mathrm{mg}$ & $1 \mathrm{mg}$ & $2 \mathrm{mg}$ \\
\hline Motilidad total (\%) & $90.98 \pm 2.35$ & $91.96 \pm 1.66$ & $92.52 \pm 2.00$ \\
Motilidad progresiva (\%) & $83.88 \pm 4.82$ & $85.32 \pm 4.41$ & $86.53 \pm 4.06$ \\
Inmóviles (\%) & $9.02 \pm 2.35$ & $8.04 \pm 1.66$ & $7.49 \pm 2.00$ \\
Vitalidad (\%) & $85.79 \pm 4.55$ & $87.23 \pm 4.51$ & $88.34 \pm 4.73$ \\
Anormalidades espermáticas (\%) & $7.50 \pm 1.38$ & $7.77 \pm 1.63$ & $8.33 \pm 1.71$ \\
HOST (\%) & $86.61 \pm 3.99$ & $87.81 \pm 3.67$ & $88.08 \pm 3.62$ \\
\hline
\end{tabular}

Sin diferencias estadísticas entre medias de cada variable

La motilidad total y progresiva después de la fase de estabilización en la etapa de refrigerado varió entre 90.98 y $92.56 \%$ y entre 83.88 y $86.53 \%$, respectivamente, sin diferencia significativa entre tratamientos (Cuadro 1). Estos resultados son superiores a los obtenidos por Spalekova et al. (2014), quienes obtuvieron motilidades totales $43 \mathrm{y}$ $63 \%$ y motilidades progresivas entre $35 \mathrm{y}$ $60 \%$ a las $24 \mathrm{~h}$ de incubación por efecto de la cafeína con concentraciones entre 0 y $4 \mathrm{mmol} / \mathrm{l}$. Por otro lado, Cabrera et al. (2010) reportaron porcentajes de motilidad cercanos al presente estudio ( 84.6 y $83.3 \%$ ) al evaluar dos dilutores (Tris - Fructosa Yema de huevo y Citrato - Glucosa - Yema de huevo) en carneros Assaf.

Los porcentajes de vitalidad (85.79$88.34 \%$ ) obtenidos fueron ligeramente superiores a los reportados por Apaza (2017) al evaluar dilutores a base de diferentes tipos de yema de huevo; no obstante, dicho autor encontró menores porcentajes de anormalidades en el semen refrigerado de carnero. Por otro lado, los resultados de integridad de membrana fueron ligeramente superiores a los observados por Cabrera y Pantoja (2008) al evaluar el efecto de los dilutores Tris y Ovine Freezing.
A pesar de no haber diferencia significativa entre tratamientos, los resultados siguen la tendencia observada en el estudio de Salmon et al. (2016), quienes encontraron que el esperma tratado con $2 \mathrm{mg}$ de CLC/120 x $10^{6}$ espermatozoides ofrece una mayor protección a la calidad del esperma (motilidad y vitalidad) después del shock frío en los espermatozoides de carnero.

\section{Viabilidad del Semen Criopreservado}

Los porcentajes de espermatozoides móviles totales, móviles progresivos, vivos y con membrana citoplasmática intacta fueron significativamente superiores cuando las muestras fueron tratadas con metil- $\beta$ ciclodextrina cargada de colesterol previamente a la criopreservación comparado con el tratamiento control $(\mathrm{p}<0.05)$, siendo la concentración de $2 \mathrm{mg}$ de CLC/ $120 \times 10^{6}$ de espermatozoides (T3) la dosis de mejor comportamiento (Cuadro 2). La frecuencia de anormalidades espermáticas no fue afectada por los tratamientos en estudio.

T3 presentó 9.93\% más de motilidad total y $6.7 \%$ más de espermatozoides móviles progresivos respecto al grupo control (T1). Las ventajas obtenidas en T3 concuerdan con 
Cuadro 2. Efecto de la adición de metil- $\beta$-ciclodextrina saturada de colesterol (CLC) sobre las características microscópicas pos-descongelación de semen de carnero (CLC/120 × $10^{6}$ espermatozoides)

\begin{tabular}{lccc}
\hline Variable & $0 \mathrm{mg}$ & $1 \mathrm{mg}$ & $2 \mathrm{mg}$ \\
\hline Motilidad total (\%) & $65.34 \pm 5.94^{\mathrm{b}}$ & $68.68 \pm 5.54^{\mathrm{b}}$ & $75.27 \pm 5.7^{\mathrm{a}}$ \\
Motilidad progresiva (\%) & $55.13 \pm 7.59^{\mathrm{b}}$ & $56.07 \pm 7.83^{\mathrm{b}}$ & $61.83 \pm 7.12^{\mathrm{a}}$ \\
Inmóviles (\%) & $34.66 \pm 5.94^{\mathrm{a}}$ & $31.32 \pm 5.54^{\mathrm{a}}$ & $24.73 \pm 5.7^{\mathrm{b}}$ \\
Vitalidad (\%) & $56.58 \pm 7.34^{\mathrm{b}}$ & $60.70 \pm 8.01^{\mathrm{ab}}$ & $66.23 \pm 7.99^{\mathrm{a}}$ \\
Anormalidades espermáticas (\%) & $9.60 \pm 1.99^{\mathrm{a}}$ & $10.13 \pm 1.94^{\mathrm{a}}$ & $11.23 \pm 1.96^{\mathrm{a}}$ \\
HOST (\%) & $50.54 \pm 7.17^{\mathrm{b}}$ & $56.49 \pm 7.43^{\mathrm{b}}$ & $63.71 \pm 5.9^{\mathrm{a}}$ \\
\hline
\end{tabular}

${ }^{a, b}$ Letras diferentes dentro de filas muestran diferencias significativas entre las medias $(p<0.05)$

los resultados positivos obtenidos por Batissaco (2014) en semen de carnero y con Moraes et al. (2010) en semen de toro. No obstante, Purdy et al. (2010) no encontraron diferencias importantes en términos de motilidad total y motilidad progresiva con el uso de CLC en semen de toros. Las diferencias entre estudios pueden atribuirse a la especie animal, a las características de espermatozoides de diferentes individuos dentro de una especie o líneas dentro de una especie que pueden responder de manera diferente a la CLC (Mocé et al., 2010b). Además, dentro de los factores responsables de esta variabilidad de resultados se debe considerar que en estos experimentos se usaron diferentes diluyentes y temperaturas de incubación con las CLC, así como diversos protocolos de refrigeración y congelación.

El porcentaje promedio de vitalidad fue superior en T3 $(\mathrm{p}<0.05)$, resultado similar al obtenido por Motamedi et al. (2014), quienes obtuvieron $10 \%$ extra de vialidad espermática con la adición de $3 \mathrm{mg}$ de CLC/ $120 \times 10^{6} \mathrm{de}$ células en el diluyente Tris en semen de carnero. Las evidentes efectos protectores del colesterol en las membranas espermáticas, reflejadas en mayores porcentajes de vitalidad, se deben al efecto del colesterol sobre la estabilización de la membrana y la disminución de la permeabilidad de la membrana, que permite interacciones entre células, además de proporcionar microambientes químicos y físicos adecuados, siendo un antioxidante de membrana (Crockett, 1998). Por otro lado, el ligero incremento de anormalidades espermáticas, sin diferencias entre tratamientos, es de esperarse al pasar por el proceso de criopreservación (Verstegen et al., 2002).

La mayor protección de la funcionalidad de la membrana espermática se observó en T3 ( $\mathrm{p}<0.05)$, encontrándose $13.2 \%$ de diferencia porcentual con relación al tratamiento control. Este resultado fue superior al grado de protección obtenido por Salmon (2016) de $29.6 \%$ en el semen suplementado a base de leche desnatada + CLC ( 2 mg de CLC/120 x $10^{6}$ espermatozoides) y de $21 \%$ en el semen control diluido con Triladyl ${ }^{\circledR}$. Por otro lado, Mocé et al. (2010b) obtuvieron mayores diferencias con semen de carnero tratado con $2 \mathrm{mg}$ de CLC/ $120 \times 10^{6}$ de espermatozoides previo a la congelación que en el grupo control no suplementado, posiblemente debido a que los carneros empleados se caracterizaban por su baja respuesta a la congelación, mientras que en el presente estudio eran carneros cuyo semen era de buena motilidad luego del proceso de congelación/descongelación. Así mismo, habría que considerar que, si bien, la adición de la dosis $2 \mathrm{mg}$ de CLC/120 x $10^{6}$ de espermatozoides mejora la integri- 
dad de membrana, al mismo tiempo, los resultados son menores si la concentración excede esta concentración (Mocé et al., 2010b).

Por medio del uso de ciclodextrinas, es posible manipular el contenido de colesterol celular, cambiando las condiciones que van desde el enriquecimiento neto de colesterol hasta el agotamiento; además, otorga un método apropiado, preciso y reproducible para modificar el contenido de colesterol de células (Christian et al., 2007).

El tratamiento de los espermatozoides de carnero con CLC puede aumentar el contenido de colesterol de los espermatozoides en más de 1.9 veces y aunque se tienen pérdidas en el enfriamiento y la criopreservación, el contenido de colesterol se conserva en mayor proporción en los espermatozoides tratados con CLC respecto de aquellos no tratados (Mocé et al., 2010a). Así, la adición de CLC a los diluyentes mejora la viabilidad de los espermatozoides y reduce el daño del acrosoma posteriores a la congelación/descongelación (Tomás et al., 2014).

\section{Conclusiones}

- La adición de metil- $\beta$-ciclodextrina saturada de colesterol previo a la criopreservación conservó con mayor efectividad la motilidad, vitalidad e integridad de membrana acrosomal del semen de carnero luego de la descongelación.

- Los mejores resultados en viabilidad espermática pos-descongelación fueron obtenidos con la adición de $2 \mathrm{mg}$ de CLC/120 millones de espermatozoides.

\section{Literatura Citada}

1. Anel L, Boixo JC, Anel E, Carbajo M, Dominguez JC, Olmedo JA, Melcon C. 1992. Fertility of churra ewes following intrauterine insemination by laparoscopy with frozen-thawed semen. Proc $12^{\text {th }}$ Int. Cong. Anim. Reprod. \& A.I. The Hague, The Netherlands.

2. Apaza L. 2017. Efecto protector de yema de huevo en la viabilidad de semen congelado de carnero. Tesis de Médico Veterinario y Zootecnista. Puno, Perú: Univ. Nacional del Altiplano. 140 p.

3. Batissaco L. 2014. Criopreservação do sêmen ovino com incorporação de colesterol por ciclodextrina. Tesis de Master. São Paulo: Univ. de São Paulo. $113 \mathrm{p}$.

4. Cabrera P, Pantoja C. 2008. Influencia de los dilutores tris y ovine freezing sobre la integridad de la membrana citoplasmática durante la congelación de semen de ovinos en pajillas de $0.5 \mathrm{ml}$. Rev Inv Vet Perú 19: 152-159. doi: 10.15381/rivep.v19i2.1162

5. Cabrera P, Orellana J, Pantoja $C$. 2010. Efecto de dos dilutores sobre la motilidad e integridad de la membrana espermática en semen congelado de ovinos. Rev Inv Vet Perú 21: 154-160. doi: 10.15381/rivep.v21i2.130

6. Christian AE, Haynes MP, Phillips MC, Rothblat GH. 2007. Use of cyclodextrins for manipulating cellular cholesterol content. J Lipid Res 38: 22622272.

7. Correa JR, Zavos PM. 1994. The hypoosmotic swelling test: its employment as an assay to evaluate the functional integrity of frozen-thawed bovine sperm membrane. Theriogenology 42: 351-360. doi: 10.1016/0093691x(94)90280-1

8. Crockett EL. 1998. Cholesterol function in plasma membranes from ectotherms: membrane-specific roles in adaptation to temperature. Integr Comp Biol 38: 291 304. doi: $10.1093 / \mathrm{icb} / 38.2 .291$

9. Farshad A, Amidi A, Koohi A, Rashidi A. 2001. Effect of cholesterol-loadedcyclodextrin in presence and absence of egg yolk during freezing step on quality of Markhoz buck's spermatozoa. Asian Austral J Anim 24: 181-189. doi: 10.5713/ ajas.2011.10141 
10. Holt WV. 2000. Fundamental aspects of sperm cryobiology: the importance of species and individual differences. Theriogenology 53: 47-58. doi: 10.1016/ S0093-691X(99)00239-3

11. Mocé E, Blanch E, Tomás C, Graham $J K .2010 a$. Use of cholesterol in sperm cryopreservation: present moment and perspectives to future. Reprod Domest Anim 45: 57-66. doi: 10.1111/j.14390531.2010.01635.x

12. Mocé E, Purdy PH, Graham JK. 2010b. Treating ram sperm with cholesterol-loaded cyclodextrins improves cryosurvival. Anim Reprod Sci 118: 236-247. doi: 10.1016/j.anireprosci.2009.06.013

13. Moore AI, Squires EL, Graham JK. 2005. Adding cholesterol to the stallion sperm plasma membrane improves cryosurvival. Cryobiology 51: 241-249. doi: 10.1016/j.cryobiol.2005.07.004

14. Moraes EA, Graham JK, Torres CA, Meyers M, Spizziri B. 2010. Delivering cholesterol or cholestanol to bull sperm membranes improves cryosurvival. Anim Reprod Sci 118: 148-154. doi: 10.1016/ j.anireprosci.2009.08.002

15. Motamedi-Mojdehi R, Roostaei-Ali Mehr M, Rajabi-Toustani R. 2014. Effect of different levels of glycerol and cholesterol-loaded cyclodextrin on cryosurvival of ram spermatozoa. Reprod Domest Anim 49: 65-70. doi: 10.1111/rda.12225

16. Purdy PH, Graham JK. 2004. Effect of cholesterol-loaded cyclodextrin on the cryosurvival of bull sperm. Cryobiology 48: 36-45. doi: 10.1016/j.cryobiol.2003.12.001
17. Purdy PH, Mocé E, Stobart R, Murdoch WJ, Moss GE, Larson B, Ramsey S, et al. 2010. The fertility of ram sperm held for $24 \mathrm{~h}$ at $5{ }^{\circ} \mathrm{C}$ prior to cryopreservation. Anim Reprod Sci 118: 231-235. doi: 10.1016/j.anireprosci.2009.06.014

18. Quispe F. 1998. Efecto de dos dilutores, tiempo de equilibrio y raza sobre la motilidad de semen descongelado de ovino. Tesis de Magíster. Lima: Univ. Nacional Agraria La Molina. 73 p.

19. Salmon VM, Castonguay F, DemersCaron V, Leclerc P, Bailey JL. 2016. Cholesterol-loaded cyclodextrin improves ram sperm cryoresistance in skim milkextender. Anim Reprod Sci 177:1-11. doi: 10.1016/j.anireprosci.2016.11.011

20. Spaleková E, Makarevich A, Kubovikova E, Ostró A, Chrenek P. 2014. Effect of caffeine on functions of cooling-stored ram sperm in vitro. Acta Vet Brno 83: 19-25. doi: 10.2754/avb201483010019

21. Tomás C, Gómez-Fernández J, Gómez-Izquierdo E, Mocé E, de Mercado E. 2014. Addition of cholesterolloaded cyclodextrins to the thawing extender: effects on boar sperm quality. Reprod Domest Anim 49: 427-432. doi: 10.1111/rda.12291

22. Verstegen J, Iguer-Ouada M, Onclin K. 2002. Computer assisted semen analyzers in andrology research and veterinary practice. Theriogenology 57 : 149-179. doi: 10.1016/S0093-691X(01)00664-1

23. Watson P. 2000. The causes of reduced fertility with cryopreserved semen. Anim Reprod Sci 60-61: 481-492. doi: 10.1016/ S0378-4320(00)00099-3 